\title{
Modelagem da precipitação pluvial diária intra-anual da Bacia Hidrográfica Paraná III associada aos eventos ENOS
}

\author{
Antonio L. Baú ${ }^{1}$, Carlos A. V. de Azevedo² \& Adriano de A. Bresolin ${ }^{3}$
}

\begin{abstract}
RESUMO
A região oeste do estado do Paraná, que abrange a Bacia Hidrográfica Paraná III, possui um sistema agrícola ainda muito dependente das condições climáticas. Diante disto, os aspectos que envolvem a construção de modelos probabilísticos, capazes de determinar parâmetros de ocorrência e quantificação da precipitação pluvial diária, têm-se destacado como fator necessário e fundamental para consecução dos projetos regionais, em todas as áreas. Portanto, o objetivo deste trabalho foi realizar a construção dos referidos modelos considerando-se a ocorrência dos fenômenos ENOS e se utilizando, para tal, os dados de 34 estações meteorológicas com séries superiores a 21 anos de registros diários, obtidos junto à Agência Nacional de Águas. A modelagem da ocorrência da precipitação diária foi tratada por meio dos processos de Markov enquanto a modelagem da quantidade precipitada foi feita por meio do ajuste à função gama de probabilidade. Para a validação do ajuste utilizou-se o teste de Kolmogorov-Smirnov e a partir dos resultados obtidos foi possível constatar que a metodologia desenvolvida pode ser aplicada para a simulação de séries sintéticas de precipitação pluvial diária para a área de estudo.
\end{abstract}

Palavras-chave: recursos hídricos, séries sintéticas, distribuição gama de probabilidade

\section{Modeling of daily intra-annual precipitation of the Paraná III Basin associated with events ENSO}

\begin{abstract}
The western region of Paraná state, covering the Paraná III Basin, has an agricultural system still largely dependent on climatic conditions. Thus, the aspects that involve the construction of probabilistic models, able to determine parameters of occurrence and quantification of the daily precipitation have emerged as a necessary and fundamental factor for the achievement of regional projects in all areas. Therefore, the objective of this work was the construction of such models considering the occurrence of ENSO phenomena, using for this purpose data from 34 weather stations with series of more than 21 years of daily records, which were obtained from the ANA (Agência Nacional de Águas). The modeling of the occurrence of daily precipitation was treated through the Markov processes, while the modeling of the precipitated amount was performed by adjustment of the gamma function of probability. For validation of the adjustment, the Kolmogorov-Smirnov test was used. From the results it was established that this methodology can be applied to the simulation of synthetic series of daily rainfall for the study area.
\end{abstract}

Key words: water resources, synthetics series, gamma probability distribution

${ }^{1}$ UTFPR/SELIB. Av. Brasil, 4232, Parque Independência, CEP 85884-000, Medianeira, PR. Fone: (45) 3240-8050. E-mail: albau@utfpr.edu.br ${ }^{2}$ UAEA/UFCG. Rua Aprígio Veloso, 882, Bairro Universitário, CEP 58429-140, Campina Grande, PB. Fone: (83) 3310-1056 e (83) 310-1318. E-mail: cazevedo@deag.ufcg.edu.br

${ }^{3}$ UTFPR/SEDUP. Av. Brasil, 4232, Parque Independência, CEP 85884-000, Medianeira, PR. Fone: (45) 3240-8141. E-mail: aabresolin@utfpr.edu.br 


\section{INTRODUÇÃO}

A relação, extremamente dependente entre o clima e a agricultura, conduz a estudos sobre o comportamento climático, sobretudo estudos mais abalizados sobre a precipitação com o objetivo de estabelecer certezas e definir parâmetros para uma relação precipitação-produtividade agrícola mais eficaz.

Com o propósito de simular a precipitação pluvial, modelos estocásticos têm sido propostos em pesquisas relativas à área para geração de séries de precipitações visto serem de grande utilidade para diversas aplicações práticas, dentre as quais se destacam a determinação de cheias de projeto para obras hidráulicas, a análise da viabilidade econômica para implementação de irrigação em dado local, as análises referentes a culturas agrícolas e aos estudos relacionados à hidroeletricidade. Ademais, a aplicação cada vez mais habitual do Método de Monte Carlo para estimar as propriedades estatísticas das variáveis hidrológicas envolvidas agrega uma importância extra para a geração de séries consistentes (Castellví et al., 2001; Calgaro et al., 2009; Detzel \& Mine, 2011).

Wan et al. (2005), Martin et al. (2007) e Semenov (2008) afirmam que modelos estocásticos aplicados em hidrologia são, muitas vezes, utilizados para complementar dados climatológicos observados diariamente e que também podem ser usados para gerar séries longas de dados sintéticos para análise de risco. Eles se concentram nas variações dos parâmetros meteorológicos do dia-a-dia transformando fluxos de números aleatórios produzidos por algoritmos computacionais em sequências de valores baseados em várias propriedades estatísticas das observações climatológicas atuais. Além disso, esses modelos têm sido amplamente utilizados para avaliar, a longo prazo, os prováveis efeitos antropogênicos nas mudanças climáticas.

Deste modo, os modelos de Markov são frequentemente propostos para se obter rapidamenteas previsões do tempo "estados" em algum momento futuro, através de informação favorecida pelo estado atual. Uma das aplicações da cadeia de Markov é a modelagem da ocorrência de precipitação diária (Sharif et al., 2007; Damé et al., 2007; Selvaraj \& Selvi, 2010).

Conforme Souza Júnior et al. (2009) as variáveis meteorológicas, estando relacionadas a fenômenos de escala global, também sofrem influências do fenômeno ENOS (El Niño - Oscilação Sul) que, por sua vez, está relacionado a anomalias oceânicas e atmosféricas na região equatorial do Oceano Pacífico. Acredita-se que o fenômeno ENOS seja um dos responsáveis pelas flutuações globais do clima, dentre todos os fenômenos atmosféricos, sendo que ele continua sendo o mais polêmico até os dias atuais, dada à sua escala global de abrangência e sua extensa durabilidade temporal.

Neste contexto o presente estudo teve como objetivo a análise dos aspectos que envolvem a construção de modelos probabilísticos, capazes de determinar parâmetros de ocorrência e quantificação da precipitação pluvial no âmbito da Bacia Hidrográfica Paraná III, considerando-se a ocorrência do fenômeno ENOS.

\section{Material e Métodos}

As séries históricas de registros diários de precipitação foram obtidas junto à Agência Nacional de Águas (ANA), por meio do seu endereço eletrônico de suporte HIDROWEB, em arquivos de dados no formato MS Access ${ }^{\circledR}$, nos quais constavam registros já devidamente consistidos e atualizados.

A partir desses registros procedeu-se, através de rotinas de programação, à leitura detida dos dados, à detecção de dados faltantes e ao referido tamanho das séries.

Após a análise e seleção das estações que possuíam maior série de dados, maior quantidade de séries de dados completas e posição espacial compatível com a cobertura total da bacia hidrográfica, foram selecionadas as séries históricas de precipitação de 34 estações pluviométricas considerandose, como data limite de registros, o dia 31/12/2010. O tamanho das séries selecionadas foi determinado em função da existência e da qualidade dos dados e de forma a conter, minimamente, elementos meteorológicos com 21 anos de registros visto que, conforme salienta Bailey (1990) para um bom estudo da necessidade de irrigação de uma região devem ser analisados os elementos meteorológicos de um período de aproximadamente 20 anos. Melhor estudo seria possível notadamente com séries extensas de dados. A maior série de dados é da estação Porto Guaíra (código 2454001) com 68 anos de registros.

A área de abrangência compreende a Bacia Hidrográfica Paraná III (Figura 1) que corresponde à totalidade da área de

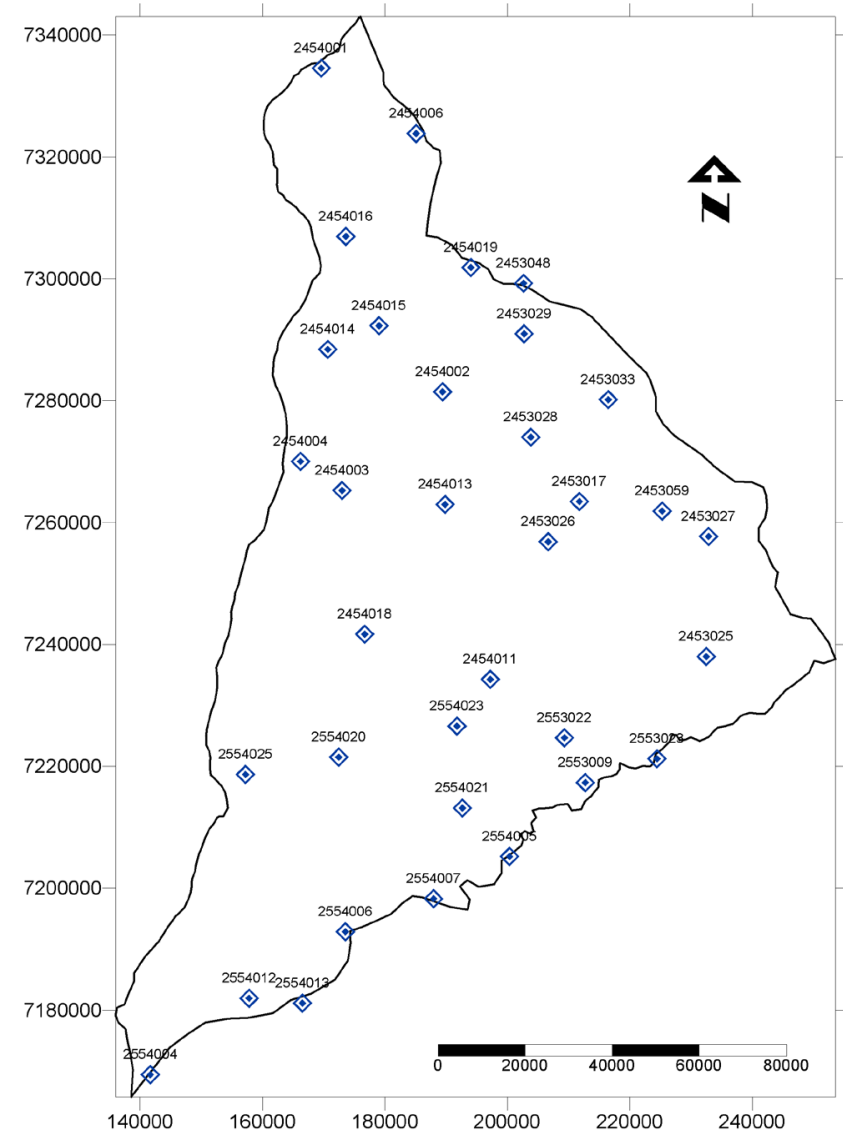

Figura 1. Representação espacial das estações pluviométricas selecionadas, Bacia Hidrográfica Paraná III 
drenagem dos afluentes pertencentes ao território paranaense os quais lançam suas águas diretamente no rio Paraná.

A precipitação pluvial diária foi descrita, nesta pesquisa, pela Eq. 1, proposta por Assis \& Villa-Nova (1994).

$$
Z_{t}=X_{t} \cdot Y_{t}
$$

em que:

$\mathrm{Z}_{\mathrm{t}} \quad$ - precipitação pluvial diária, $\mathrm{mm}$

$X_{t} \quad$ - ocorrência de chuva

$\mathrm{Y}_{\mathrm{t}} \quad$ - quantidade de chuva, $\mathrm{mm}$

De acordo com Mehrotra \& Sharma (2009) pode-se definir, como um dia chuvoso, aquele cujo valor medido chegar ao limiar de $0,3 \mathrm{~mm} \mathrm{~d}^{-1}$. Diante disto, para este trabalho considerouse $X_{t}=1$ quando a precipitação foi maior ou igual a $0,3 \mathrm{~mm}$ e $\mathrm{X}_{\mathrm{t}}=0$ para os demais casos.

A caracterização dos eventos por ano de ocorrência obedeceu aos critérios estabelecidos pela NOAA (2011) considerando-se, para tal, a predominância da ocorrência do fenômeno, ou seja, a ocorrência do maior número de meses do ano associado à intensidade relativa ao período considerado. Esta caracterização serviu de base para se definir a estratificação dos dimensionamentos, cálculos e resultados por anos de ocorrência de El Niño e La Niña, comparando-os com anos de normalidade. A Tabela 1 representa os anos de ocorrência com a respectiva classificação por evento predominante.

Tabela 1. Anos de ocorrência dos fenômenos ENOS

\begin{tabular}{cccccccc}
\hline & El Niño & \multicolumn{3}{c}{ Neutros } & \multicolumn{3}{c}{ La Niña } \\
1939 & 1969 & 1997 & 1942 & 1961 & 1938 & 1971 & 1996 \\
1940 & 1972 & 1998 & 1943 & 1967 & 1949 & 1973 & 1999 \\
1941 & 1977 & 2002 & 1944 & 1978 & 1950 & 1974 & 2000 \\
1946 & 1982 & 2003 & 1945 & 1979 & 1954 & 1975 & 2001 \\
1947 & 1983 & 2004 & 1948 & 1980 & 1955 & 1976 & 2007 \\
1951 & 1986 & 2005 & 1952 & 1981 & 1956 & 1984 & 2008 \\
1957 & 1987 & 2006 & 1953 & 1990 & 1962 & 1985 & 2010 \\
1963 & 1991 & 2009 & 1958 & 1993 & 1964 & 1988 & - \\
1965 & 1992 & - & 1959 & - & 1968 & 1989 & - \\
1966 & 1994 & - & 1960 & - & 1970 & 1995 & - \\
\hline
\end{tabular}

Fonte: NOAA (2011)

Para determinação da ocorrência da precipitação foi utilizado no presente trabalho, o modelo matemático baseado na cadeia de Markov, admitindo-se a hipótese da persistência de primeira ordem e dois estados, ou seja, hipótese que considera a condição de que o evento atual depende do evento ocorrido no dia anterior para os estados chuvoso e seco. Para tanto, as cadeias markovianas de primeira ordem consideram hipóteses de combinação entre os estados seco (0) e chuvoso (1) do seguinte modo:

$\mathrm{P}_{00} \quad$ - probabilidade de dia atual seco e anterior seco

$\mathrm{P}_{01}$ - probabilidade de dia atual seco e anterior chuvoso

$\mathrm{P}_{10}$ - probabilidade de dia atual chuvoso e anterior seco

$\mathrm{P}_{11}$ - probabilidade de dia atual chuvoso e anterior chuvoso

As hipóteses de combinação pressupõem a determinação das probabilidades de transição entre estados por meio de uma matriz considerada matriz de transição (MT).

$$
\mathrm{MT}=\left|\begin{array}{ll}
\mathrm{P}_{00} & \mathrm{P}_{01} \\
\mathrm{P}_{10} & \mathrm{P}_{11}
\end{array}\right|
$$

Os elementos da matriz foram, portanto, determinados pela relação entre o número $(\mathrm{N})$ de ocorrências das combinações de dias (secos/chuvosos) das séries históricas, por estação pluviométrica, da forma como segue:

$$
\begin{gathered}
\mathrm{P}_{00}=\frac{\mathrm{N}_{00}}{\mathrm{~N}_{00}+\mathrm{N}_{10}} \\
\mathrm{P}_{01}=\frac{\mathrm{N}_{01}}{\mathrm{~N}_{01}+\mathrm{N}_{11}}=1-\mathrm{P}_{11} \\
\mathrm{P}_{10}=\frac{\mathrm{N}_{10}}{\mathrm{~N}_{00}+\mathrm{N}_{10}}=1-\mathrm{P}_{00} \\
\mathrm{P}_{11}=\frac{\mathrm{N}_{11}}{\mathrm{~N}_{01}+\mathrm{N}_{11}}
\end{gathered}
$$

em que:

$\mathrm{N}_{00}$ - número de dias secos com anterior também seco

$\mathrm{N}_{01}$ - número de dias secos com anterior chuvoso

$\mathrm{N}_{10}$ - número de dias chuvosos com anterior seco

$\mathrm{N}_{11}$ - número de dias chuvosos com dia anterior chuvoso

Para a referida modelagem das séries sintéticas de ocorrência da precipitação pluvial diária foram determinados os valores relativos à probabilidade de transição para cada um dos novos estados das séries que, em síntese, assumiram os valores complementares de $\mathrm{P}_{10}$. Por meio de uma rotina computacional estabeleceu-se uma análise comparativa entre as probabilidades determinadas $\left(\mathrm{P}_{00}\right.$ e $\left.\mathrm{P}_{10}\right)$ e os números aleatórios uniformemente distribuídos $\left(\mathrm{A}_{(0,1)}\right)$ gerados em um intervalo entre 0 e 1, conforme explicita o fluxograma da Figura 2.

Para determinação da quantidade de precipitação pluvial diária adotou-se, conforme Cho et al. (2004), Liao et al. (2004) e Castellvíet al. (2004), o modelo probabilístico teórico

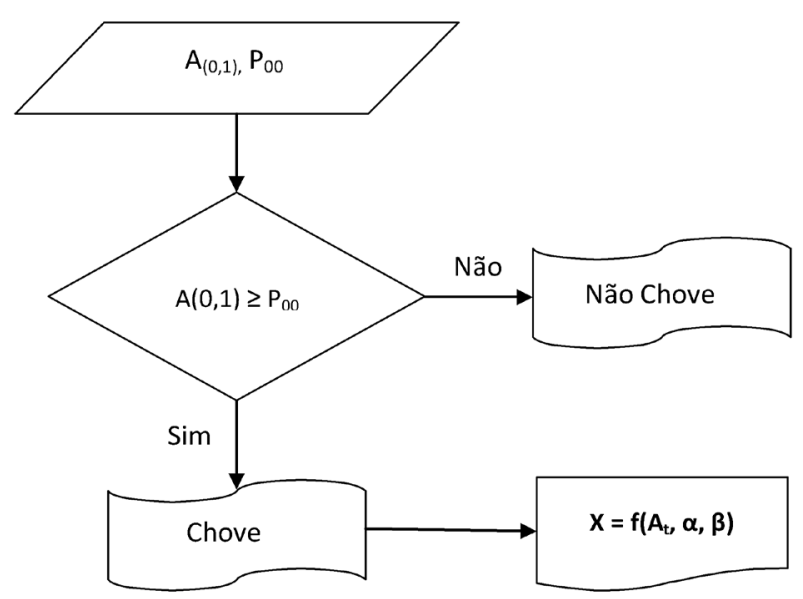

Figura 2. Fluxograma do processo de modelagem da precipitação pluvial. Adaptado de Calgaro et al. (2009) 
gama de distribuição de probabilidade cuja função densidade de distribuição acumulada a dois parâmetros $(\alpha$ e $\beta)$ é:

$$
f(x, \alpha, \beta)=\frac{x^{1-\alpha} e^{\frac{-x}{\beta}}}{\beta^{\alpha} \Gamma(\alpha)}
$$

em que:

x - quantidade diária de precipitação

$\Gamma \quad$ - função gama

O parâmetro $\alpha$ é adimensional, geralmente inferior a um e, principalmente, considera os casos de pequenas quantidades de precipitação. $O$ parâmetro $\beta$ tem a mesma unidade da precipitação. Ambos os parâmetros estão relacionados com a precipitação diária esperada $\mu$, através da expressão:

$$
\mu=\alpha \beta
$$

Comumente, o parâmetro $\beta$ é maior que 1 quando a quantidade de precipitação é expressa em milímetros e leva em conta ventos de chuvas intensas. Os parâmetros $\alpha$ e $\beta$ da distribuição gama de probabilidade, necessários para esta modelagem, foram determinados pelo método da máxima verossimilhança, sendo:

$$
\begin{gathered}
\alpha=\frac{1}{4 y}\left(1+\sqrt{1+\frac{4 y}{3}}\right) \\
\beta=\frac{\bar{X}}{\alpha} \\
y=\ln \bar{X}-\frac{1}{n} \sum_{j=1}^{n} \ln x_{j}
\end{gathered}
$$

em que:

$\mathrm{X}$ - média precipitada no período

$\mathrm{x}_{\mathrm{j}}$ - quantidade de chuva no evento

$\mathrm{n}$ - tamanho da amostra

Para validação da referida modelagem foram realizados testes de adequação de ajustes dos dados com o auxílio de modelos de testes não paramétricos de Kolmogorov-Smirnov, com nível de significância de 0,05 de probabilidade.

Como meio complementar de análise utilizou-se o p-valor que nos testes de hipótese pode ser considerado como a probabilidade de uma amostra ser extraída de uma população para o caso da hipótese nula $\left(\mathrm{H}_{0}\right)$ ser verdadeira.

Tanto para o teste de Kolmogorov-Smirnov como para o teste p-valor foram utilizados os dados diários estimados e os dados observados de precipitação diária para cada uma das estações estratificadas por fenômeno ENOS.

\section{Resultados E Discussão}

Observa-se, na Figura 3, que os valores médios das probabilidades de transição $\mathrm{P}_{00}, \mathrm{P}_{01}, \mathrm{P}_{10}$ e $\mathrm{P}_{11}$ para os anos de evento El Niños e comportaram de modo que os maiores valores de média para $\mathrm{P}_{10}$ se concentraram nos períodos de $1^{\circ}$ e $4^{\circ}$ trimestres enquanto para $P_{00}$, a concentração ocorreu nos períodos de $2^{\circ}$ e $3^{\circ}$ trimestres. Para as Probabilidades $\mathrm{P}_{01}$ e $\mathrm{P}_{11}$ não se observou concentração de valores de média em nenhum período, especificamente. Os valores médios das probabilidades de transição para os anos de evento La Niñas se comportaram de modo semelhante aos anos de evento El Niño. Para os anos neutros a concentração dos maiores valores de média de $\mathrm{P}_{00}$ e $\mathrm{P}_{11}$ ocorreu nos $2^{\circ}$ e $3^{\circ}$ trimestres, ao passo que para $\mathrm{P}_{10}$ a concentração ocorreu nos $1^{\circ}$ e $4^{\circ}$ trimestres. A probabilidade de transição $\mathrm{P}_{01}$ não demonstrou concentração de maiores ou menores valores de média em período específico. A Figura 3 demonstra, também, os níveis das probabilidades médias mensais de transição, de todas as estações, para os anos de fenômenos ENOS podendo-se denotar que a probabilidade de não chover hoje, visto que não choveu ontem $\left(\mathrm{P}_{00}\right)$ é maior que as demais probabilidades.

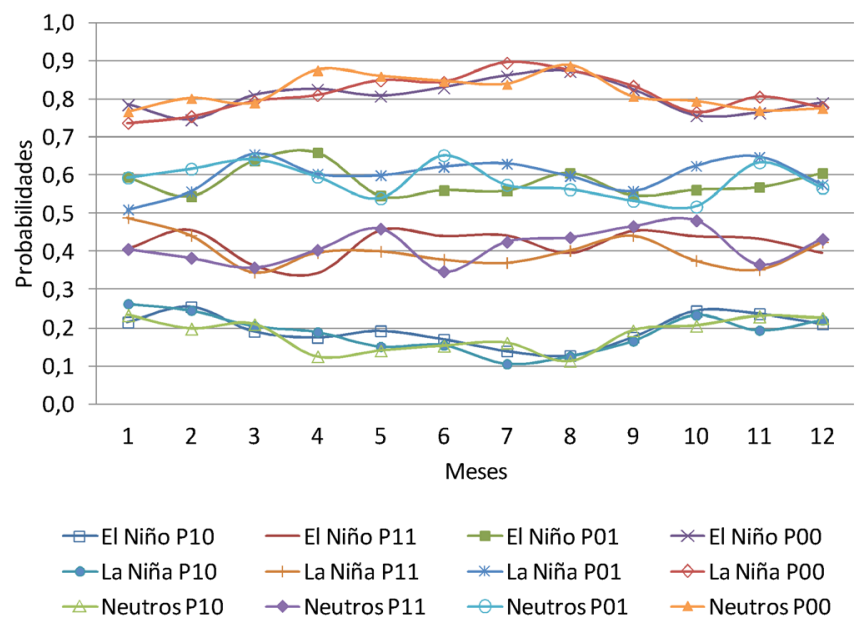

Figura 3. Probabilidades médias de transição $P_{00^{\prime}}$ $\mathrm{P}_{10}, \mathrm{P}_{01}$ e $\mathrm{P}_{11}$ dos anos de El Niño, La Niña e neutros

As Figuras 4A, B e C têm o propósito de estabelecer termo comparativo entre os parâmetros de Markov das séries sintéticas e das séries históricas para os fenômenos de escala El Niño, La Niña e Neutros. Para tanto observa-se, de modo geral, que as séries simuladas (sintéticas) reproduzem as séries observadas (originais). $\mathrm{O}$ propósito de apresentar apenas $\mathrm{P}_{10}$ e $\mathrm{P}_{00}$ é de evitar exposição demasiada de elementos gráficos visto que os resultados são similares para as outras probabilidades de transição.

Constatou-se, também, que não houve variação significativa de probabilidade entre os meses do ano. Deste modo e do ponto de vista da análise, a utilização dos valores médios mensais não implicou em erros no processo de simulação.

Ante o exposto pode-se afirmar que a exploração da natureza do processo markoviano demonstrou que uma Cadeia de Markov de primeira ordem e dois estados fornece uma descrição adequada da ocorrência de precipitação diária, constatação que também foi confirmada por Selvaraj \& Selvi (2010), Martins et al. (2011) e Wan et al. (2005).

Para consecução das análises necessárias determinaram-se os momentos estatísticos dos parâmetros $\alpha$ e $\beta$, de acordo com a Tabela 2, abaixo representada. 
A.

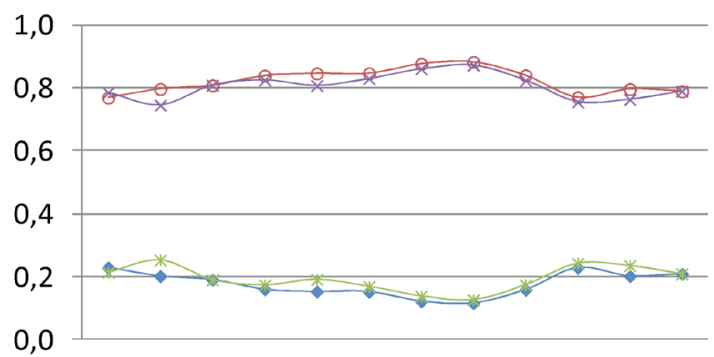

B.

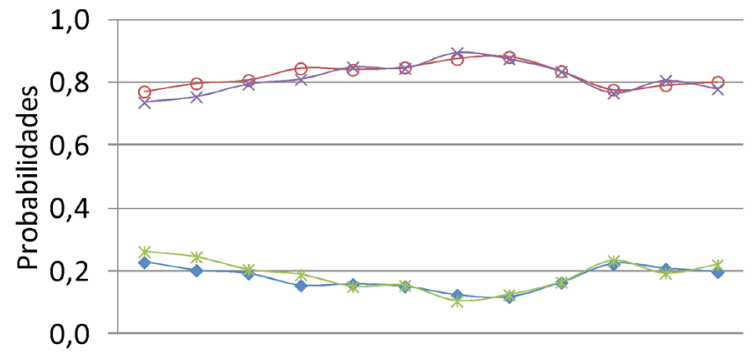

C.

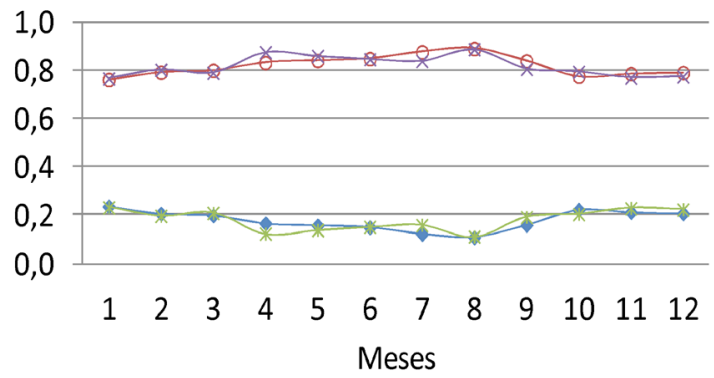

$\rightarrow$ Sintética P10 $\rightarrow$-Sintética PO0 $\rightarrow$ * Original P10 $*$ Original POO

Figura 4. Probabilidades médias de transição $\left(\mathrm{P}_{10} \mathrm{e}\right.$ $P_{00}$ ) das séries originais e sintéticas para os eventos El Niño (A) e La Niña (B) e para os anos neutros (C)

Os valores do parâmetro $\alpha$ acusaram, para os doze meses do conjunto de anos que corresponde à ocorrência do evento El Niño, coeficiente de variação média de $19,67 \%$ determinando, deste modo, uma classificação de média dispersão ou variabilidade dos valores.
Pode-se observar, ainda, que os menores valores relativos ao parâmetro $\alpha$ ocorreram nos meses de menor precipitação pluvial, constatação corroborada por Botelho \& Morais (1999) e Moreira et al. (2010).

Por meio dos dados constantes na Tabela 2 constata-se que o coeficiente de variação média dos valores do parâmetro $\beta$, para os doze meses dos anos de ocorrência do evento El Niño, foi de 18,39\% determinando, desta maneira, uma classificação de média dispersão e que tanto os valores menores como os limites maiores ocorreram nos meses de menor precipitação pluvial.

Os valores de $\alpha$ para os anos de predominância do fenômeno La Niña, demonstraram uma amplitude de variação relativamente pequena e acusaram, para os doze meses, coeficiente de variação média de $16,85 \%$ determinando, deste modo, uma classificação de média dispersão ou variabilidade dos dados.

O coeficiente de variação média dos valores do parâmetro $\beta$ para os doze meses dos anos de ocorrência do La Niña, foi de $13,41 \%$ determinando, assim, uma classificação de média dispersão constatando-se também que os valores determinantes dos limites máximos ocorreram nos meses de julho e novembro e os limites mínimos nos meses de abril, agosto e novembro não demonstrando, portanto, tendência preferencial em relação à ocorrência de precipitação para períodos mensais característicos de precipitação predominante. Comportamento semelhante para os valores de $\beta$ foi encontrado por Moreira et al. (2010).

Os valores de $\alpha$ para os anos de neutralidade demonstraram uma amplitude de variação relativamente pequena, com exceção apenas para o mês de agosto.

Com o aumento do valor de $\alpha$ o grau de assimetria da distribuição diminui, o que significa que esta exceção está pressupostamente relacionada com a baixa pluviosidade registrada para as séries relativas ao mês de agosto dos anos de neutralidade. O coeficiente de variação média acusou $17,60 \%$ determinando, então, uma classificação de média dispersão ou variabilidade dos valores ao passo que os limites máximos e mínimos dos valores do parâmetro $\alpha$ não demonstraram tendência preferencial de ocorrência.

Tabela 2. Momentos estatísticos mensais dos parâmetros $\alpha$ e $\beta$ da função gama das 34 estações pluviométricas

\begin{tabular}{|c|c|c|c|c|c|c|c|c|c|c|c|c|c|c|}
\hline \multirow{9}{*}{$\alpha$} & & & Jan & Fev & Mar & $\overline{A b r}$ & Mai & Jun & Jul & $\overline{\text { Ago }}$ & Set & Out & Nov & $\overline{D e z}$ \\
\hline & & Média & 1,05 & 1,02 & 1,04 & 1,08 & 1,07 & 1,05 & 1,23 & 1,07 & 1,15 & 1,14 & 1,29 & 1,07 \\
\hline & El Niño & $\begin{array}{c}\text { SD } \\
\text { CV (\%) }\end{array}$ & $\begin{array}{c}0,17 \\
16,05\end{array}$ & $\begin{array}{c}0,20 \\
19,35\end{array}$ & $\begin{array}{c}0,19 \\
18,66\end{array}$ & $\begin{array}{c}0,25 \\
22,93\end{array}$ & $\begin{array}{c}0,21 \\
19,69\end{array}$ & $\begin{array}{c}0,18 \\
17,45\end{array}$ & $\begin{array}{c}0,30 \\
24,37\end{array}$ & $\begin{array}{c}0,23 \\
21,45\end{array}$ & $\begin{array}{c}0,23 \\
20,19\end{array}$ & $\begin{array}{c}0,23 \\
19,86\end{array}$ & $\begin{array}{c}0,27 \\
20,82\end{array}$ & $\begin{array}{r}0,16 \\
15,19\end{array}$ \\
\hline & & Média & 1,12 & 1,12 & 1,11 & 1,16 & 1,10 & 1,05 & 1,04 & 1,09 & 1,15 & 1,14 & 1,13 & 1,09 \\
\hline & La Niña & SD & 0,19 & 0,22 & 0,22 & 0,31 & 0,24 & 0,22 & 0,23 & 0,18 & 0,21 & 0,23 & 0,28 & 0,20 \\
\hline & & CV (\%) & 17,25 & 19,94 & 19,87 & 26,34 & 22,21 & 20,66 & 22,53 & 16,85 & 17,99 & 20,40 & 24,74 & 18,53 \\
\hline & & Média & 1,02 & 1,06 & 1,18 & 1,07 & 1,04 & 1,23 & 1,12 & 1,11 & 1,04 & 1,11 & 1,12 & 1,04 \\
\hline & Neutros & SD & 0,18 & 0,30 & 0,33 & 0,24 & 0,21 & 0,38 & 0,31 & 0,53 & 0,22 & 0,25 & 0,20 & 0,23 \\
\hline & & CV (\%) & 17,82 & 28,02 & 28,09 & 22,58 & 20,55 & 31,01 & 27,74 & 47,59 & 20,89 & 22,32 & 17,60 & 22,07 \\
\hline \multirow{8}{*}{$\beta$} & & Média & 19,27 & 19,38 & 18,01 & 22,91 & 26,48 & 20,50 & 15,07 & 16,46 & 18,68 & 21,27 & 18,92 & 20,14 \\
\hline & El Niño & $\begin{array}{c}\text { SD } \\
\text { CV (\%) }\end{array}$ & $\begin{array}{c}3,13 \\
16,22\end{array}$ & $\begin{array}{c}2,99 \\
15,44\end{array}$ & $\begin{array}{c}3,05 \\
16.93\end{array}$ & $\begin{array}{c}3,75 \\
16.37\end{array}$ & $\begin{array}{c}5,12 \\
1934\end{array}$ & $\begin{array}{r}2,45 \\
1196\end{array}$ & $\begin{array}{c}6,61 \\
43,84\end{array}$ & $\begin{array}{c}3,22 \\
1956\end{array}$ & $\begin{array}{c}2,66 \\
14,24\end{array}$ & $\begin{array}{c}3,78 \\
1776\end{array}$ & $\begin{array}{c}3,04 \\
16,06\end{array}$ & $\begin{array}{c}2,61 \\
12,95\end{array}$ \\
\hline & & Média & 16,75 & 16,41 & 16,29 & 19,71 & 16,85 & 16,91 & 16,68 & 14,55 & 15,30 & 19,80 & 18,75 & $\begin{array}{l}19,04 \\
\end{array}$ \\
\hline & La Niña & SD & 2,65 & 2,94 & 2,84 & 4,16 & 3,37 & 3,43 & 5,25 & 2,41 & 2,29 & 3,17 & 5,24 & 2,55 \\
\hline & & CV (\%) & 15,80 & 17,90 & 17,44 & 21,11 & 20,00 & 20,25 & 31,51 & 16,58 & 14,99 & 15,99 & 27,96 & 13,41 \\
\hline & & Média & 17,26 & 15,05 & 13,40 & 24,16 & 26,80 & 13,20 & 15,24 & 20,85 & 20,24 & 20,07 & 18,05 & 21,86 \\
\hline & Neutros & SD & 3,66 & 3,72 & 3,36 & 7,87 & 4,79 & 3,49 & 3,83 & 4,96 & 3,03 & 3,53 & 3,79 & 3,72 \\
\hline & & CV (\%) & 21,24 & 24,69 & 25,06 & 32,59 & 17,86 & 26,43 & 25,10 & 23,80 & 14,96 & 17,61 & 20,98 & 17,02 \\
\hline
\end{tabular}

* SD - Desvio padrão e CV - Coeficiente de variação 
O coeficiente de variação médio dos valores do parâmetro $\beta$ para os doze meses foi de $14,96 \%$ determinando, desta maneira, uma classificação de média dispersão.

Os anos neutros apresentaram os limites máximos e mínimos dos valores do parâmetro $\beta$ (parâmetro de escala). De acordo com Semenov (2008) o parâmetro $\beta$ indica o grau de dispersão entre os dados de determinada série, razão pela qual o referido parâmetro leva em conta eventos de chuvas intensas. Observouse, portanto, que os valores de $\beta$ para a grande maioria dos anos El Niño, foram maiores que os valores de $\beta$ para os anos de evento La Niña. Esta constatação está associada à ocorrência de maior pluviosidade nos anos de El Niño; o contrário pode ser constatado com o comportamento do parâmetro $\beta$, para os anos de fenômeno La Niña.

Para o parâmetro $\alpha$ (parâmetro de forma) os valores máximos e mínimos ocorreram nos anos de eventos El Niño enquanto para o parâmetro $\beta$ (parâmetro de escala) se deram nos anos neutros, conforme se observa nas Figuras $5 \mathrm{~A}$ e $\mathrm{B}$.

A.

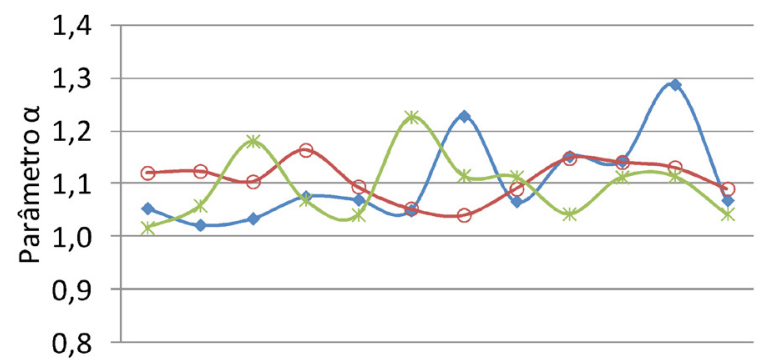

B.

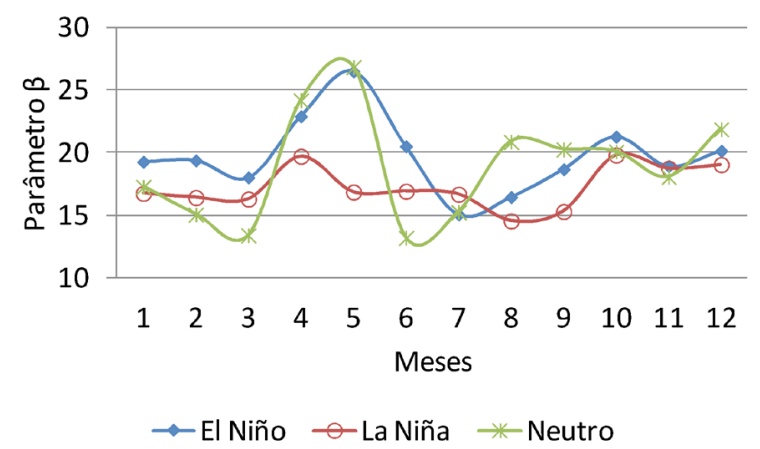

Figura 5. Valores médios mensais dos parâmetros $\alpha$ (A) e $\beta$ (B) em função dos fenômenos ENOS

O conjunto de valores médios do parâmetro $\alpha$ acusou coeficientes de variação (Figura 6A) menores para os anos de La Niña determinando, deste modo, maior homogeneidade do respectivo conjunto de valores. A sazonalidade e a transição intra-anual dos eventos determinaram, de modo consistente, a diferença de coeficientes de variação do parâmetro $\alpha$ entre os fenômenos. Considerando sugestão de intervalos de classificação proposto por Botter et al. (1996) que determina para $\mathrm{CV}<15 \%$ baixa variabilidade, conclui-se que os valores do referido parâmetro para as médias mensais dos anos relativos aos 3 fenômenos, demonstraram baixa variabilidade.

$\mathrm{Na}$ Figura 6B observa-se que os valores médios do parâmetro $\beta$ acusaram coeficientes de variação menores para os anos de La Niña determinando, então, maior homogeneidade do respectivo conjunto de valores. A sazonalidade e a transição intra-anual dos eventos determinaram, consistentemente, a diferença de coeficientes de variação dos valores médios do parâmetro $\beta$ entre os fenômenos. Para os dados relativos aos anos de fenômenos El Niño e La Niña, o conjunto de valores pode ser classificado de baixa variabilidade; no entanto, para os anos de neutralidade os dados se caracterizaram por apresentar média variabilidade no seu conjunto.

A.

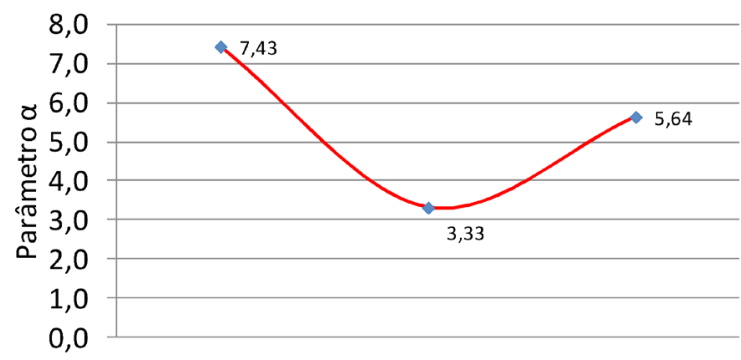

B.

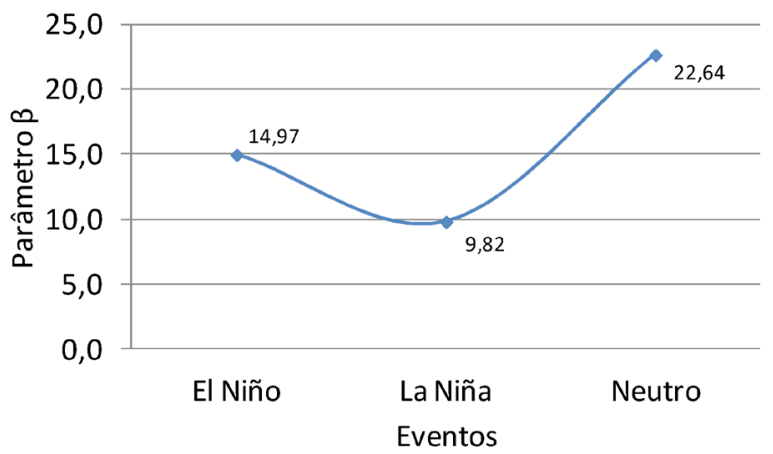

Figura 6. Coeficiente de variação dos valores médiosdos parâmetros $\alpha$ (A) e $\beta$ (B) da função gama - eventos ENOS

Os parâmetros variam consideravelmente, mês a mês, dentro de uma estação pluviométrica, mesmo se considerando a estratificação por fenômeno ENOS, com o que se presume seja influenciado por razões como a sazonalidade, o regime de chuvas mais ou menos intenso e ocasional, a quantidade de precipitação para determinado dia em relação à média diária mensal e à localização da estação.

Para o efeito intra-anual do fenômeno ENOS no regime pluviométrico da Bacia Hidrográfica Paraná III e se considerando a média das 34 estações, observou-se um incremento na ordem de 7,63\% na precipitação total média anual para os anos de eventos El Niño enquanto em eventos La Niña houve um decréscimo de 5,63\% na precipitação total média anual, em relação aos anos de neutralidade.

Durante eventos de El Niño os meses que acusaram maior precipitação total foram maio e abril, constatando-se um incremento de chuva em nove dos doze meses do ano. Em períodos de La Niña a maior precipitação se concentrou nos meses de outubro e abril com decréscimo da lâmina precipitada em 4 dos 12 meses do ano, quais sejam: abril, maio, agosto e setembro.

Observa-se, portanto, que os resultados deste trabalho indicam a existência de uma relação direta entre a precipitação ocorrida na Bacia Hidrográfica Paraná III e os índices de anomalias associados aos eventos ENOS em que, para os anos caracterizados por eventos El Niño, o incremento de 
precipitação média, quando estratificado por período decenal, demonstra tendência de aumento em todas as estações fato este também observado por Alexander et al. (2006).

Foi possível constatar que existe uma forte relação dos fenômenos El Niño e La Niña com os episódios de intensidade e frequência da precipitação na Bacia Hidrográfica Paraná III haja vista que a análise dos resultados demonstrou que o comportamento da precipitação diária manteve padrão de quantidade e ocorrência simultaneamente ao surgimento dos referidos fenômenos.

A bacia apresenta, em períodos de ocorrência dos fenômenos ENOS, variabilidade pluviométrica espacial e temporal, de que os anos de máximos e mínimos são considerados, em sua maioria, anos relacionados a tais fenômenos.

A pluviosidade na área de abrangência deste estudo (34 estações) varia sob a tutela das escalas de tempo interanual e intra-anual. É provável que na escala de tempo interanual se possa constatar maior influência dos fenômenos ENOS nos valores de precipitação máxima e mínima da Bacia; todavia, é possível denotar, também, na escala intra-anual, tal influência, supostamente com menor amplitude visto que os fenômenos ENOS têm caráter de ocorrência interanual (duração de 6 a 18 meses).

Por fim e uma vez concluídas as análises necessárias, pode-se inferir que os fenômenos climatológicos globais, como exemplo do ENOS (El Niño - Oscilação Sul) e até mesmo de âmbito regional, exercem influência sobre o regime de chuvas da Bacia Hidrográfica Paraná III.

Conforme se denota nas Figuras 7A, 7B e 7C, as séries de precipitação média determinaram, visualmente, uma excelente qualidade de ajuste, que pode ser constatada por meio dos valores relativos aos coeficientes de determinação $\left(\mathrm{R}^{2}\right)$ na análise de regressão linear proposta.

A análise dos coeficientes de variação constante na Tabela 3 , demonstrou a existência de grande dispersão entre os dados médios das séries diárias mensais; entretanto, manteve certa equivalência entre os coeficientes de variação das séries originais e das séries sintéticas. A grande dispersão demonstrada pelos
A.

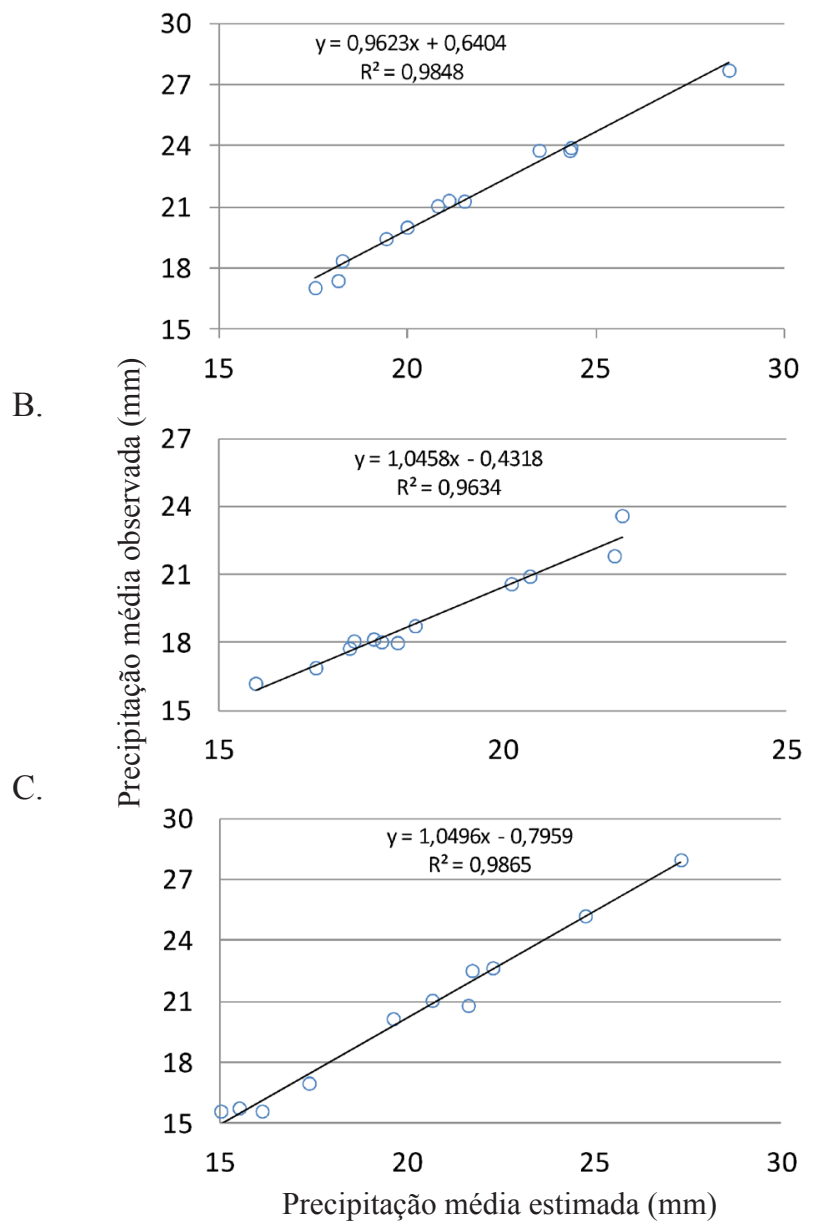

Figura 7. Valores mensais de precipitação média diária $(\mathrm{mm})$ observados versus estimados para os eventos El Niño (A), La Niña (B) e os anos neutros (C)

coeficientes de variação das referidas séries, provavelmente relacionada à ocorrência de chuvas intensas, também determina o fato do desvio padrão apresentar comportamento semelhante ao da média, fato também observado por Gomes \& Cruz (2002).

Tabela 3. Momentos estatísticos mensais dos valores médios da precipitação diária das 34 estações - eventos El Niño, La Niña e neutros

\begin{tabular}{|c|c|c|c|c|c|c|c|c|c|c|c|c|c|c|}
\hline \multirow{10}{*}{ 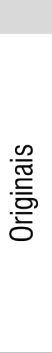 } & & & Jan & Fev & Mar & Abr & Mai & Jun & Jul & Ago & Set & Out & Nov & Dez \\
\hline & & Média & 20,03 & 19,47 & 18,39 & 23,93 & 27,72 & 21,34 & 17,41 & 17,07 & 21,08 & 23,78 & 23,80 & 21,30 \\
\hline & El Niño & con & 20,85 & 21,65 & 20,22 & 24,61 & 27,14 & 21,26 & 19,06 & 16.92 & 20,26 & 23,30 & 20,53 & \\
\hline & & CV (\%) & 104,11 & 111,20 & $\begin{array}{l}109,98 \\
\end{array}$ & 102,84 & 97,91 & 99,61 & 109,43 & 99,11 & 96,14 & 97,98 & 86,27 & $\begin{array}{l}102,92 \\
\end{array}$ \\
\hline & & Média & 19,98 & 19,42 & 18,25 & 24,33 & 28,52 & 21,08 & 18,14 & 17,53 & 20,79 & 24.30 & 23,48 & 21,50 \\
\hline & La Niña & SD & 19,25 & 18,69 & 17,92 & 23,52 & 27,18 & 19,39 & 15,76 & 16,57 & 19,34 & 22,47 & 20,66 & 20,13 \\
\hline & & CV (\%) & 96,37 & 96,24 & 98,19 & 96 & 95,29 & 91,98 & 86 & 94,51 & 93,05 & 92 & 9 & 93,63 \\
\hline & & Média & 18,45 & 18,14 & 17,72 & 21, & 17,86 & 17,37 & 16,70 & 15,64 & 17,30 & 22,09 & 20,14 & 20,47 \\
\hline & Neutros & SD & 19,26 & 18,19 & 17,79 & 20,5 & 17,79 & 17,37 & 17,34 & 15,40 & 16,76 & 21,02 & 19,25 & 20,14 \\
\hline & & CV (\%) & 104,38 & 100,26 & 100,40 & 93 , & 99 , & 98 & 103,80 & 98,49 & 96,88 & 95,15 & 5 & 98,36 \\
\hline \multirow{9}{*}{ 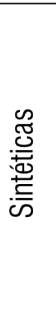 } & & Média & 18,75 & 18,01 & 18,17 & 21,84 & 18,05 & 18,08 & 16,90 & 16,21 & 17,76 & 23,61 & 20,60 & 20,93 \\
\hline & El Niño & SD & 17,3 & 16 & 17,03 & 20 & 16,69 & 17,31 & 15 & 15,15 & 15 & 2 & & 19,74 \\
\hline & & CV (\%) & 92,49 & 91,79 & 93,77 & 94,62 & 92,48 & 95,74 & 92,62 & 93,42 & 87,82 & 93,10 & 46 & 94,29 \\
\hline & & Média & 17,37 & 15,21 & 15,02 & 24,75 & 27,31 & 15,51 & 16,12 & 21,63 & 20,66 & 21,73 & & 22,28 \\
\hline & La Niña & SD & 18,50 & 16,67 & 14,65 & 25,28 & 27,06 & 15,22 & 15,82 & 21,96 & 21,05 & 21,32 & 19,11 & 22,92 \\
\hline & & CV (\%) & 106,47 & 109,56 & 97,58 & 102,13 & 99,12 & 98,13 & 98,16 & 101,56 & 101,88 & 98,13 & 97,39 & 102,86 \\
\hline & & Média & 17,00 & 14,93 & 15,62 & 25,22 & $\begin{array}{l}27,98 \\
\end{array}$ & 15,78 & 15,63 & 20,82 & 21,07 & 22,53 & 20,17 & 22,67 \\
\hline & Neutros & SD & 15 & 13,98 & 14 & 23,44 & 26,16 & 13,02 & 14 & 19,38 & 19,11 & 21,56 & 18,53 & 22,11 \\
\hline & & CV (\%) & 93,82 & 93,66 & 91,58 & 92,93 & 93,47 & 82,51 & 92,03 & 93,10 & 90,71 & 95,66 & 91,89 & 97,50 \\
\hline
\end{tabular}


Tabela 4. Resultados do teste de Kolmogorov-Smirnov para os eventos ENOS

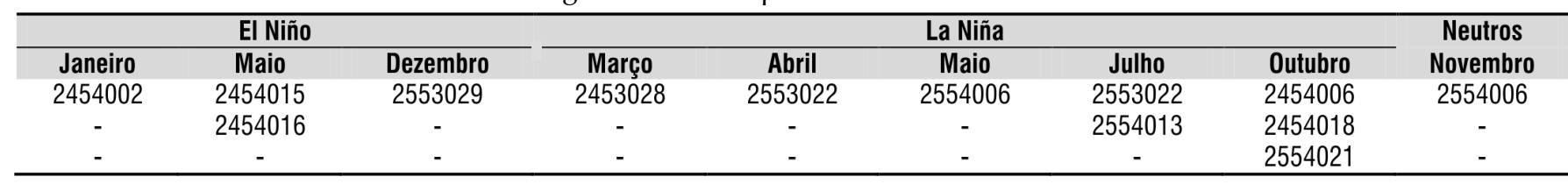

A rotina computacional desenvolvida para determinar a qualidade da aderência dos dados de precipitação (séries originais $\mathrm{x}$ séries sintéticas) retornou os valores correspondentes a $\mathrm{H}_{0}(0$ e 1 , respectivamente, para aceitação e rejeição da hipótese nula $\mathrm{H}_{0}$ ), $\mathrm{p}$-valor (para p-valor $>0,05$ a hipótese nula pode ser considerada verdadeira) e o valor de Kolmogorov (k) que corresponde ao Dmáx.

Analisando os referidos dados constatou-se, para os anos de eventos El Niño, La Niña e neutros, que ocorreram rejeições da hipótese $\mathrm{H}_{0}$, para as estações e meses descritos na Tabela 4 e que equivalem, respectivamente, a 0,98, 1,96 e $0,25 \%$ das séries de precipitação média que não se ajustaram rigorosamente à distribuição gama de probabilidade. As demais séries de todas as estações se ajustaram à função densidade de distribuição gama de probabilidade. Deste modo, para os níveis percentuais apresentados pode-se considerar que a rejeição não é significativa e que o teste de Kolmogorov-Smirnov e o teste p-valor, atenderam à referida hipótese, isto é, a hipótese de igualdade das amostras para o nível de significância desejado.

Com relação ao ajuste das séries de precipitação pluvial diária, conclui-se que a função gama de probabilidade foi mais eficaz para os anos de neutralidade, supostamente em função da menor ocorrência de eventos extremos de precipitação nos referidos anos. Observou-se também que os meses de maior incidência de precipitação pluvial registraram melhor ajuste à distribuição, constatação corroborada por Lyra et al. (2006) e Dourado Neto et al. (2005).

Com isto, constata-se que a distribuição gama de probabilidade a dois parâmetros, foi adequada para a modelagem das séries de precipitação diária da Bacia Hidrográfica Paraná III, constatação confirmada também nos trabalhos de Cho et al. (2004), Liao et al. (2004), Murta et al. (2005), Selvaraj \& Selvi (2010) e Moreira et al. (2010).

\section{Conclusões}

1. As cadeias de Markov de primeira ordem e dois estados se mostraram adequadas para a modelagem da ocorrência da precipitação diária da Bacia Hidrográfica Paraná III.

2. A função de distribuição gama de probabilidade a dois parâmetros mostrou-se adequada para o ajuste das séries de precipitação diária de cada uma das 34 estações pluviométricas da referida bacia.

3. As séries sintéticas de precipitação pluvial diária (simuladas) mantiveram as características das séries históricas de precipitação (observadas).

4. A metodologia desenvolvida pode ser aplicada para a simulação de séries sintéticas de precipitação pluvial diária, em cada uma das 34 estações pluviométricas da referida bacia, para os eventos ENOS (El Niño - Oscilação Sul).

5. Existe uma relação direta entre a precipitação ocorrida na Bacia Hidrográfica Paraná III e os índices de anomalias associados aos eventos ENOS que, para os anos caracterizados por eventos El Niño quando estratificados por período decenal, demonstram tendência de aumento nas precipitações médias, em todas as estações.

\section{Literatura Citada}

Alexander, L. V.; Zhang, X.; Peterson, T.C.; Caesar, J.; Gleason, B.;Tank, A. M. K.; Haylock, M.; Collins, D.; Trewin, B.; Rahimzadeh, F. Tagipour, A.; Kumar, K. R.; Revadekar, J.; Griffiths, G.; Vincent, L.; Stephenson, D. B.; Burn, J.; Aguilar, E.; Brunet, M.; Taylor, M.; New, M.; Zhai, P.; I, M.; Vazquez-Aguirre, J. L. Global observed changes in daily climate extremes of temperature and precipitation. Journal UNF Geophysical Research, v.111, p.1-22,2006.

Assis, F. N. de; Villa-Nova, N. A. Modelagem da ocorrência e da quantidade de chuva em Piracicaba. Scientia Agricola, v.5, p.172-182, 1994.

Bailey, R. Irrigated crops and their management. United Kington: Farming Press Book, 1990.192p.

Botelho, V. A. V. A.; Morais, A. R. Estimativas dos parâmetros da distribuição gama de dados pluviométricos no município de Lavras, estado de Minas Gerais. Ciência e Agrotecnologia,v.23, p.697-706, 1999.

Botter, D. A.; Paula, G. A.; Leite, J. G.; Cordani, L. K. Noções de estatística.São Paulo: IME/USP, 1996.

Calgaro, M.; Robaina, A. D.; Peiter, M. X.; Bernardon, T. Variação espaço-temporal dos parâmetros para a modelagem estocástica da precipitação pluvial diária no Rio Grande do Sul. Engenharia Agrícola, v.29, p.196-206, 2009.

Castellví, F.; Mormeneo, I.; Perez, P.J. Generation of daily amounts of precipitation from standard climatic data: a case study for Argentina. Journal of Hydrology,v.289, p. 286-302, 2004.

Castellví, F.; Stockle, C. O.; Ibañez, M. Comparing a locally calibrated versus a generalized temperature generation process. Transactions of the ASAE, v.44, p.1143-1148, 2001.

Cho, H. K.; Bowman, K. P.; North, G. R. A comparison of gamma and lognormal distributions for characterizing satellite rain rates from the tropical rainfall measuring mission. Journal of Applied Meteorology, v.43, p.15861597, 2004.

Damé, R. de C. F.; Teixeira, C. F. A.; Lorensi, R. P. Simulação de precipitação com duração horária mediante o uso do modelo Bartlett-Lewis do pulso retangular modificado. Revista Brasileira de Agrociência, v.13, p.13-18, 2007.

Detzel, D. H. M.; Mine, M. R. M. Modelagem de quantidades precipitadas em escala diária: Uma análise comparativa. Revista Brasileira de Recursos Hídricos, v.16, p.101-110, 2011. 
Dourado Neto, D.; Assis, J. P. de; Timm, L. C.; Manfron, P. A.; Sparovek, G.; Martin, T. N. Ajuste de modelos de distribuição de probabilidade a séries históricas de precipitação pluvial diária em Piracicaba-SP. Revista Brasileira de Agrometeorologia, v.13, p.273-283, 2005.

Gomes, B. M.; Cruz, R. L. Comportamento espacial dos percentis 75 da precipitação decendial para o estado de São Paulo, na primavera. Revista Irriga, v.7, p.142-153, 2002.

Liao, Y; Zhang, Q.; Chen, D. Stochastic modeling of daily precipitation in China. Journal of Geographical Sciences, v.14, p.417-426. 2004.

Lyra, G. B.;Garcia, B. I. L.; Piedade, S. M. S.; Sediyama, G. C. Sentelhas, P. C. Regiões homogêneas e funções de distribuição de probabilidade da precipitação pluvial no Estado de Táchira, Venezuela. Pesquisa Agropecuária Brasileira, v.41, p.205-215. 2006.

Martin, T. N.; Storck, L.; Dourado Neto, D. Simulação estocástica da radiação fotossinteticamente ativa da temperatura do ar por diferentes métodos. Pesquisa Agropecuária Brasileira, v.42, p.1211-1219, 2007.

Martins, E. S.; Bertoni, J. C.; Clarke, R. T. Analise de precipitações diárias utilizando modelos lineares generalizados. Disponível em: <http://www.cbmet.com/ cbm-files/19-3786fb60431934bff24d592c710f4ef7.pdf > 11 Ago. 2011.

Mehrotra, R.; A. Sharma. Assessing rainfall availability over the Sydney region in a future climate using stochastic downscaling. In: World IMACS / MODSIM Congress, 18, 2009, Cairns. Proceedings... Cairns: MODSIM, p.39483954, 2009.
Moreira, P. S. P.; Dallacort, R.; Magalhães, R. A.; Inoue, M. H.; Stieler, M. C.; Silva, D. J. DA; Martins, J. A. Distribuição e probabilidade de ocorrência de chuvas no Município de Nova Maringá-MT. Revista de Ciências Agro-Ambientais, v.8, p.9-20, 2010.

Murta, R. M.; Teodoro, S. M.; Bonomo, P.; Chaves, M. A. Precipitação pluvial mensal em níveis de probabilidade pela distribuição gama para duas localidades do sudoeste da Bahia. Ciência Agrotécnicas, v.29, p.988-994, 2005.

NOAA. NOAA's El Niño Page. Disponível em < http://www. elnino.noaa.gov/>. 11 Jun. 2011.

Selvaraj, R. S.; Selvi, S.T. Stochastic modeling of daily rainfall at aduthurai. International Journal of Advanced Computer and Mathematical Sciences,v.1, p.52-57, 2010.

Semenov, M. A. Simulation of extreme weather events by a stochastic weather generator. Climate Research, v.35, p.203-212, 2008.

Sharif, M.; Burn, D. H.; Wey, K. M. Daily and Hourly Weather Data Generation using a K-Nearest Neighbour Approach. In: Canadian Hydrotechnical Conference, 2007, Winnipeg. Proceedings...Winnipeg: CHC, p.1-10, 2007.

Souza Júnior, J. A. de; Nechet, D.; Oliveira, M. C. F. de; Albuquerque, M. F. de. Estudo do comportamento da temperatura e precipitação nos períodos chuvosos e menos chuvosos em Belém-PA em anos de fortes eventos de El Niño e La Niña. Revista Brasileira de Climatologia, v.5, p.87-101, 2009.

Wan, H.; Zhang, X.; Barrow, E. M. Stocastic modeling of daily precipitation for Canada. Atmosphere-Ocean, v.43, p.23-32, 2005. 